\title{
Wall motion estimation in intracranial aneurysms
}

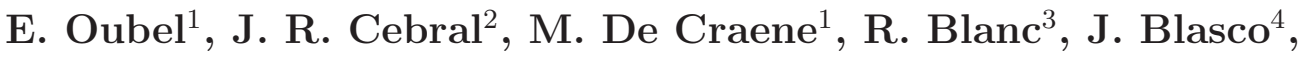
J. Macho ${ }^{4}$, C. M. Putman ${ }^{5}$ and A. F. Frangi ${ }^{1}$

${ }^{1}$ Center for Computational Imaging \& Simulation Technologies in Biomedicine (CISTIB), Information and Communication Technologies Department, Universitat Pompeu Fabra and with the Networking Center on Biomedical Research CIBER-BBN

2 Center for Computational Fluid Dynamics, Dept. of Computational and Data Sciences, George Mason University, USA

3 Department of Interventional Neuroradiology, Rothschild Foundation, Paris, France

${ }^{4}$ Department of Vascular Radiology, Hospital Clinic i Provincial de Barcelona, Barcelona, Spain

${ }^{5}$ Department of Interventional Neuroradiology, Inova Fairfax Hospital, Falls Church (VA), USA

E-mail: alejandro.frangi@upf.edu

\begin{abstract}
The quantification of wall motion in cerebral aneurysms is becoming important owing to its potential connection to rupture, and as a way to incorporate the effects of vascular compliance in computational fluid dynamics (CFD) simulations. Most of papers report values obtained with experimental phantoms, simulated images, or animal models, but the information for real patients is limited. In this paper, we have combined non-rigid registration (IR) with signal processing techniques to measure pulsation in real patients from high frame rate digital subtraction angiography (DSA). We have obtained physiological meaningful waveforms with amplitudes in the range $0 \mathrm{~mm}-0.3 \mathrm{~mm}$ for a population of 18 patients including ruptured and unruptured aneurysms. Statistically significant differences in pulsation were found according to the rupture status, in agreement with differences in biomechanical properties reported in the literature.
\end{abstract}




\section{Introduction}

Intracranial aneurysms are pathological dilations of cerebral arteries, which tend to occur at or near arterial bifurcations, mostly in the Circle of Willis (CoW). The optimal management of unruptured aneurysms is controversial and current decisionmaking is mainly based on considering their size and location, as derived from the International Study of Unruptured Intracranial Aneurysms (ISUIA) [1]. It is thought that the interaction between haemodynamics and wall mechanics plays a critical role in the formation, growth and rupture of aneurysms. The quantification of wall motion in aneurysms is becoming important since its likely connection with rupture $[2,3,4]$ and its importance for incorporating patient-specific boundary conditions in CFD simulations [5].

Many of the quantitative results of pulsation measurements reported in the literature correspond to experiments with phantoms, simulated images, or experimental models $[6,7,3,8]$. Only a few attempts of in-vivo quantification have been reported. Wardlaw et al. [9] have measured pulsation in vivo by using Power Doppler Ultrasound (PD-US), and validated their method using phantoms [10]. However, many conclusions of the study with phantoms are valid for the large changes in size considered $(42 \%$ of the cross section), but not necessary extendable for the small changes occurring in intracranial aneurysms. Meyer et al. [2] have measured aneurysmal volume changes by using Phase-Contrast Magnetic Resonance Angiography (PC-MRA). For ruptured aneurysms they obtained values of $51 \% \pm 10 \%$, which are quite large in comparison to the visual changes observed in our clinical experience. With the advent of ECG-gated 4D Computed Tomography Angiography (4D-CTA), the visualization of aneurysmal pulsation seems to have become feasible $[4,11,12]$. However, this technique presents helical motion artifacts resulting from the lack of cone beam correction during image reconstruction [13], which are visualized as a wavelike motion and produce the apparent movement of bony structures [11]. This observation and other inconsistencies about the presence of bleb pulsation have been pointed out by Matsumoto et al. [14]. These problems seem to be corrected with 64-slice CT scanners, but so far only studies of feasibility have been carried out with phantoms [6]. Recently, Zhang et al. [8] have presented a method to recover pulsation by deforming the 3D Rotational Angiography (3D-RA) rendered volume to match its projections to the $2 \mathrm{D}$ acquisitions used for the volumetric reconstruction. However, this technique has been tested only with phantom data and its application in human beings must still be proven. Table 1 summarizes the previous work in this area.

Recently, we have presented preliminary results of the application of non rigid registration techniques to recover in vivo wall motion from Digital Subtraction Angiography (DSA) sequences [15]. In this paper, we present an improved version of the method that solves some drawbacks of the initial approach. We have applied the technique to quantify wall motion in 15 patients and found a correlation between rupture and regional differences in pulsation. 
Table 1. Previous work on wall motion quantification in cerebral aneurysms. $\mathrm{R}=$ Ruptured, $\mathrm{U}=$ Unruptured; $\mathrm{WMO}=$ Wall motion occurrence; WMQ $=$ Wall motion quantification.

\begin{tabular}{llllll}
\hline Authors & Modality & Data & Status & WMO $^{\text {a }}$ & WMQ \\
\hline Hayakawa et al. $[12]$ & 4D-CTA & in vivo & All R & $4 / 23$ & no \\
Kato et al. $[4]$ & 4D-CTA & in vivo & All U & $2 / 15$ & no \\
Yaghmai et al. $[6]$ & 4D-CTA & phantom & - & - & yes \\
Boecher-S. et al. ${ }^{\mathrm{b}}[\mathrm{D}]$ & - & animal model & - & $5 / 8$ & yes \\
Wardlaw et al. $[9]$ & PD-US & in vivo & All R & $15 / 16$ & yes \\
Ueno et al. $[3]$ & PD-US & phantom & - & - & yes \\
Meyer et al. $[2]$ & PC-MRI & in vivo & $6 \mathrm{R} / 10 \mathrm{U}$ & $15 / 16$ & yes \\
Zhang et al. $[8]$ & 3D-RA & phantom & - & - & yes \\
\hline
\end{tabular}

a Wall motion occurrence is the ratio between the number of cases that present pulsation and the total number of cases.

$\mathrm{b}$ The modality is not specified since the authors used an experimental system in which the aneurysmal pulsation is measured by a laser displacement sensor.

\section{Method}

\subsection{Dataset}

Figure 1 shows the DSA sequences used in this paper. These sequences were acquired in three clinical centers: 1) Department of Interventional Neuroradiology, Inova Fairfax Hospital, 2) Department of Vascular Radiology, Hospital Clinic i Provincial de Barcelona, and 3) Department of Interventional Neuroradiology, Rothschild Foundation. At each center, an expert clinician measured neck, depth, and width of the aneurysms in the first frame using standard measuring tools of angiography scanners. To know the image resolution, the same magnitudes were measured in the 3D-RA image to avoid errors due to geometric magnification. Table 2 provides detailed information about each sequence. 


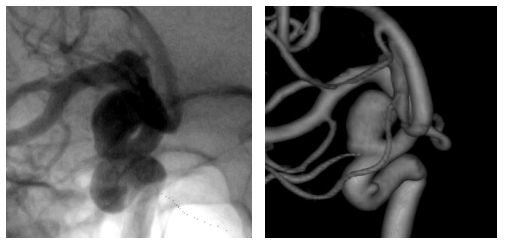

\#1

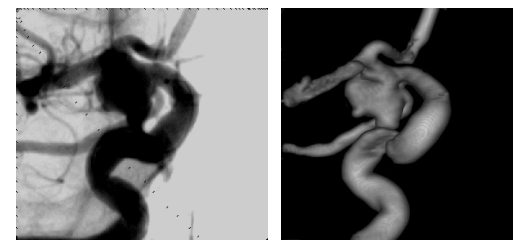

\#4

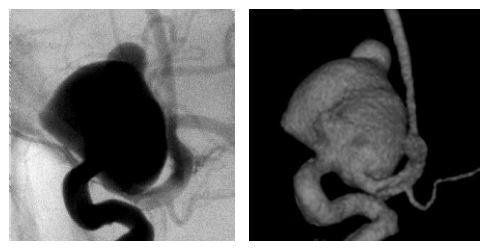

\#7

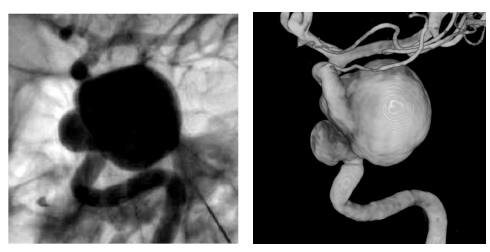

\#10

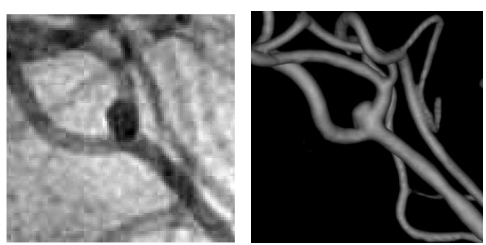

\#13

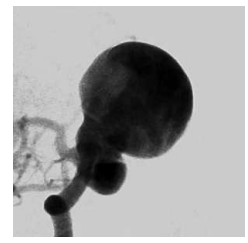

\#16

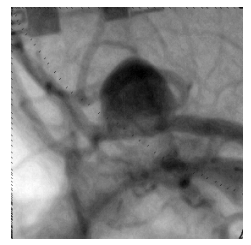

\#2

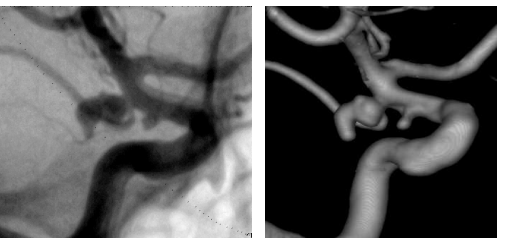

\#5

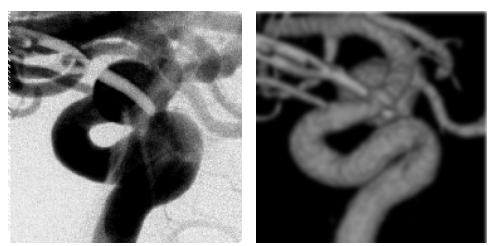

\#8
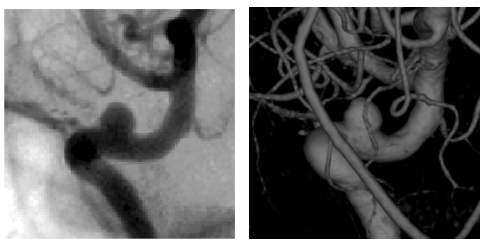

\#11

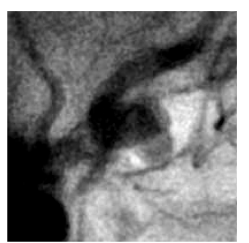

\#14
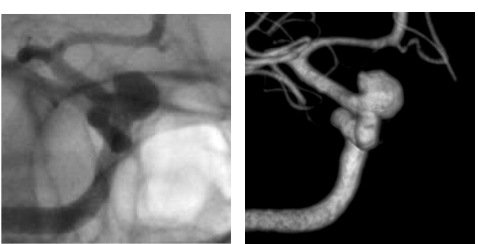

\#17

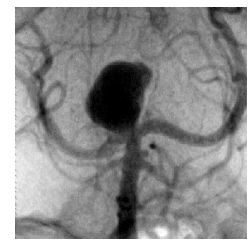

\#3

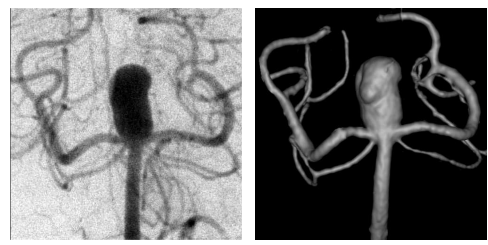

\#6

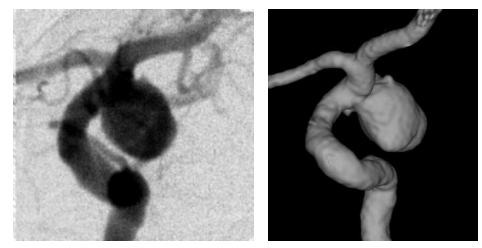

\#9
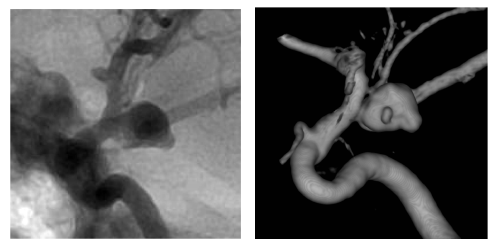

\#12

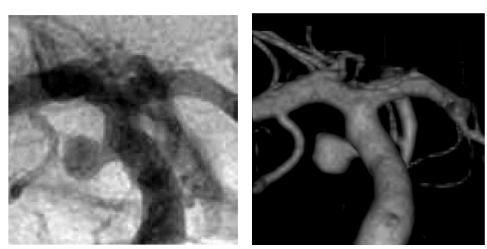

\#15
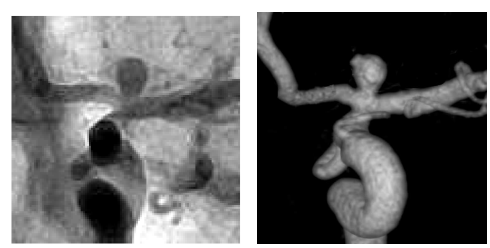

\#18

Figure 1. First frames of the DSA sequences used in this paper. The 3D-RA reconstruction is shown at right when available. 
Table 2. Table summarizing relevant information about the sequences used in this paper. M=Male, F=Female; FPS=Frames Per Second; $\mathrm{ICA}=$ Internal Carotid Artery, $\mathrm{ACA}=$ Anterior Cerebral Artery, BA=Basilar Artery, PCom=Posterior Communicating Artery, ACom=Anterior Communicating Artery, $\mathrm{MCA}=$ Middle Cerebral Artery; AR=Aspect Ratio; $\mathrm{R}=$ Ruptured, U=Unruptured; LAT=Lateral, TER $=$ Terminal; $\mathrm{Y}=$ Yes, $\mathrm{N}=\mathrm{No}, \mathrm{n} / \mathrm{a}=$ not available

\begin{tabular}{|c|c|c|c|c|c|c|c|c|c|c|c|}
\hline $\begin{array}{l}\text { Patient } \\
\#\end{array}$ & $\begin{array}{l}\text { Gender } \\
M / F\end{array}$ & $\begin{array}{l}\text { Age } \\
\text { years }\end{array}$ & Location & $\begin{array}{l}\text { Size } \\
D \times W \times N(m m)\end{array}$ & $\begin{array}{l}\text { Resolution } \\
\mu \mathrm{m} / \mathrm{pxl}\end{array}$ & $\mathrm{AR}$ & $\begin{array}{l}\text { Status } \\
\mathrm{R} / \mathrm{U}\end{array}$ & Туре & $\begin{array}{l}\text { Injection } \\
c c @ c c / s\end{array}$ & $\begin{array}{l}\text { FR } \\
F P S\end{array}$ & $\begin{array}{l}\text { Pulsatio } \\
Y / N\end{array}$ \\
\hline 1 & $\mathrm{~F}$ & 60 & ICA & $8.5 \times 6.6 \times 5.0$ & 95 & 1.7 & $\mathrm{U}$ & LAT & $9 \mathrm{cc} @ 3 \mathrm{cc} / \mathrm{s}$ & 30 & $\mathrm{Y}$ \\
\hline 2 & M & 50 & $\mathrm{ACA}$ & $7.2 \times 7.8 \times 7.8$ & 95 & 0.9 & $\mathrm{U}$ & LAT & $9 \mathrm{cc} @ 3 \mathrm{cc} / \mathrm{s}$ & 30 & $\mathrm{Y}$ \\
\hline 3 & $\mathrm{~F}$ & 47 & $\mathrm{BA}$ & $11.3 \times 9.7 \times 3.8$ & 93 & 3.0 & $\mathrm{R}$ & TER & $9 \mathrm{cc} @ 3 \mathrm{cc} / \mathrm{s}$ & 30 & $\mathrm{Y}$ \\
\hline 4 & $\mathrm{~F}$ & 78 & $\mathrm{ACM}$ & $10.0 \times 7.7 \times 5.2$ & 71 & 1.9 & $\mathrm{U}$ & TER & $9 \mathrm{cc} @ 3 \mathrm{cc} / \mathrm{s}$ & 10 & $\mathrm{~N}$ \\
\hline 5 & $\mathrm{~F}$ & 56 & ICA & $4.0 \times 4.0 \times 2.0$ & 80 & 2.0 & $\mathrm{R}$ & LAT & $9 \mathrm{cc} @ 3 \mathrm{cc} / \mathrm{s}$ & 30 & $\mathrm{~N}$ \\
\hline 6 & $\mathrm{~F}$ & 47 & $\mathrm{BA}$ & $13.0 \times 8.3 \times 5.4$ & 86 & 2.4 & $\mathrm{R}$ & TER & $24 \mathrm{cc} @ 4 \mathrm{cc} / \mathrm{s}$ & 7.5 & $\mathrm{~N}$ \\
\hline 7 & $\mathrm{~F}$ & 46 & ICA & $19.8 \times 18.9 \times 3.5$ & 88 & 5.7 & $\mathrm{R}$ & LAT & $24 \mathrm{cc} @ 4 \mathrm{cc} / \mathrm{s}$ & 3 & $\mathrm{Y}$ \\
\hline 8 & M & 28 & ICA & $9.0 \times 8.8 \times \mathrm{n} / \mathrm{a}$ & 74 & $\mathrm{n} / \mathrm{a}$ & $\mathrm{U}$ & TER & $24 \mathrm{cc} @ 4 \mathrm{cc} / \mathrm{s}$ & 2 & $\mathrm{Y}$ \\
\hline 9 & $\mathrm{~F}$ & 71 & ICA & $12.2 \times 9.2 \times 2.9$ & 97 & 4.2 & $\mathrm{R}$ & TER & $24 \mathrm{cc} @ 4 \mathrm{cc} / \mathrm{s}$ & 7.5 & $\mathrm{Y}$ \\
\hline 10 & $\mathrm{~F}$ & 41 & ICA & $17.1 \times 19.8 \times 9.9$ & 208 & 1.7 & $\mathrm{U}$ & LAT & $15 \mathrm{cc} @ 4 \mathrm{cc} / \mathrm{s}$ & 30 & $\mathrm{Y}$ \\
\hline 11 & $\mathrm{~F}$ & 51 & ICA & $4.2 \times 3.5 \times 3.0$ & 117 & 1.4 & $\mathrm{U}$ & LAT & $15 \mathrm{cc} @ 4 \mathrm{cc} / \mathrm{s}$ & 30 & $\mathrm{~N}$ \\
\hline 12 & $\mathrm{~F}$ & 37 & PCom & $7.2 \times 6.2 \times 2.4$ & 135 & 3.0 & $\mathrm{R}$ & TER & $15 \mathrm{cc} @ 4 \mathrm{cc} / \mathrm{s}$ & 60 & $\mathrm{Y}$ \\
\hline 13 & $\mathrm{~F}$ & 37 & $\mathrm{MCA}$ & $2.0 \times 2.9 \times 2.8$ & 115 & 0.7 & $\mathrm{U}$ & TER & $15 \mathrm{cc} @ 4 \mathrm{cc} / \mathrm{s}$ & 60 & $\mathrm{~N}$ \\
\hline 14 & M & 54 & ACom & $7.1 \times \times 3.3$ & 210 & 2.2 & $\mathrm{R}$ & LAT & $15 \mathrm{cc} @ 4 \mathrm{cc} / \mathrm{s}$ & 30 & $\mathrm{Y}$ \\
\hline 15 & M & 21 & PCom & $5.2 \times 5.0 \times 1.9$ & 148 & 2.7 & $\mathrm{U}$ & LAT & $15 \mathrm{cc} @ 4 \mathrm{cc} / \mathrm{s}$ & 60 & $\mathrm{~N}$ \\
\hline 16 & $\mathrm{~F}$ & 75 & ICA & $27.6 \times 30.0 \times 10.6$ & 195 & 2.6 & $\mathrm{U}$ & LAT & $15 \mathrm{cc} @ 4 \mathrm{cc} / \mathrm{s}$ & 30 & $\mathrm{Y}$ \\
\hline 17 & $\mathrm{~F}$ & 44 & ICA & $8.2 \times 10.2 \times 6.6$ & 252 & 1.2 & $\mathrm{U}$ & LAT & $15 \mathrm{cc} @ 4 \mathrm{cc} / \mathrm{s}$ & 30 & $\mathrm{~N}$ \\
\hline 18 & M & 46 & ICA & $4.2 \times 4.0 \times 2.5$ & 283 & 1.7 & $\mathrm{U}$ & TER & $15 \mathrm{cc} @ 4 \mathrm{cc} / \mathrm{s}$ & 60 & $\mathrm{~N}$ \\
\hline
\end{tabular}




\subsection{Wall motion estimation}

The method applied for quantifying pulsation consists of three main steps described next (Figure 2).

2.2.1. Image registration. A DSA study consists of an image sequence $I(\mathbf{x}, t)=$ $I_{0}(\mathbf{x}) \cdots I_{n-1}(\mathbf{x})$ of $n$ phases that provides the voxel intensity at spatial position $\mathbf{x}$ and time $t$. To quantify temporal changes in the magnitude of interest, it is necessary to establish a dense point correspondence between images. This was performed by registering each frame $I_{t}(\mathbf{x})$ to the first one $I_{0}(\mathbf{x})$, yielding a set of transformations $\mathcal{T}=\left\{\mathbf{T}_{i}(\mathbf{x})\right\}_{i=1: n}$ that provide such correspondence. In a previous paper [15], we applied an Optical Flow (OF) method [16] for IR since this type of methods are preferred for recovering small magnitude changes [17]. However, OF methods are quite sensitive to variations in intensity due to quantum noise [18] and inhomogeneities in the contrast distribution, and non-smooth deformation fields are obtained. To obtain a smoother deformation field, in this paper we have used Free-Form Deformations with B-Spline interpolation functions, and Mutual Information (MI) as metric.

Mutual information (MI) is a concept from information theory and expresses the amount of information that one image A contains about a second image B. This similarity measure was independently introduced into medical image registration by Collignon [19] and Viola [20] and is defined as

$$
M I(A, B)=H(A)+H(B)-H(A, B)
$$

where $H(A)$ and $H(B)$ are the marginal entropies of $\mathrm{A}$ and $\mathrm{B}$, and $H(A, B)$ their joint entropy. MI is particularly suited in cases where the intensity relationship of the images is unknown, which is exactly the case of DSA sequences.

B-Spline-based Free-Form Deformations (FFD) is a type of transformation used initially in computer graphics for deforming objects [21, 22] and introduced later on in medical imaging by Rueckert et al [23]. The basic idea of this type of transformation is to deform an image by manipulating an underlying mesh of control points, whose displacements are propagated to the image by using B-Spline functions. The resulting displacement field is smooth $\left(C^{2}\right.$-continuous), which is a desired property when modeling biological deformations. The main advantage of B-Splines with respect to thin-plate splines [24] and elastic-body splines [25], is their compact support that allows to constrain spatially the deformation.

2.2.2. Point propagation and feature quantification. We have analyzed temporal changes in aneurysm depth $(d(t))$, aneurysm width $(w(t))$, and artery diameter $(a(t))$. These variables were measured by taking the euclidean distance between pair of points placed in the first frame, and propagated over time with the set of transformations $\mathcal{T}$. The points used to calculate the artery diameter were placed far away from the aneurysm to have a different condition of the vessel wall at the measurement point, and to avoid the influence of aneurysmal deformations. This is important for quantification 


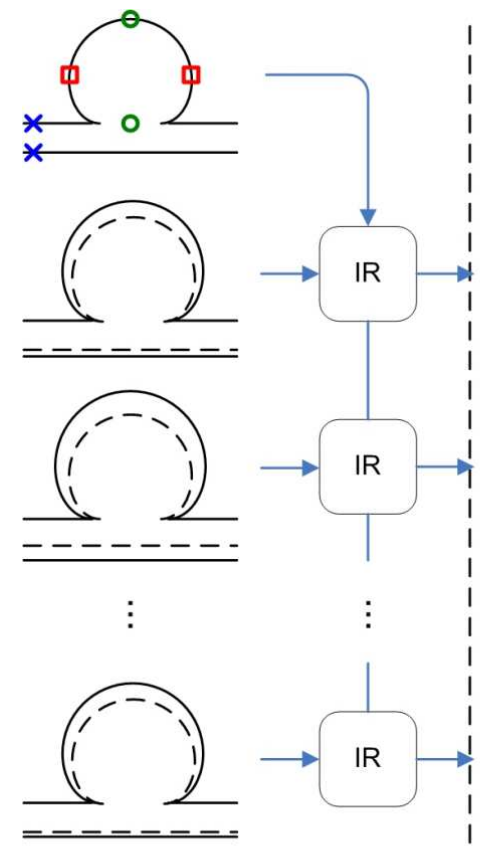

(1)

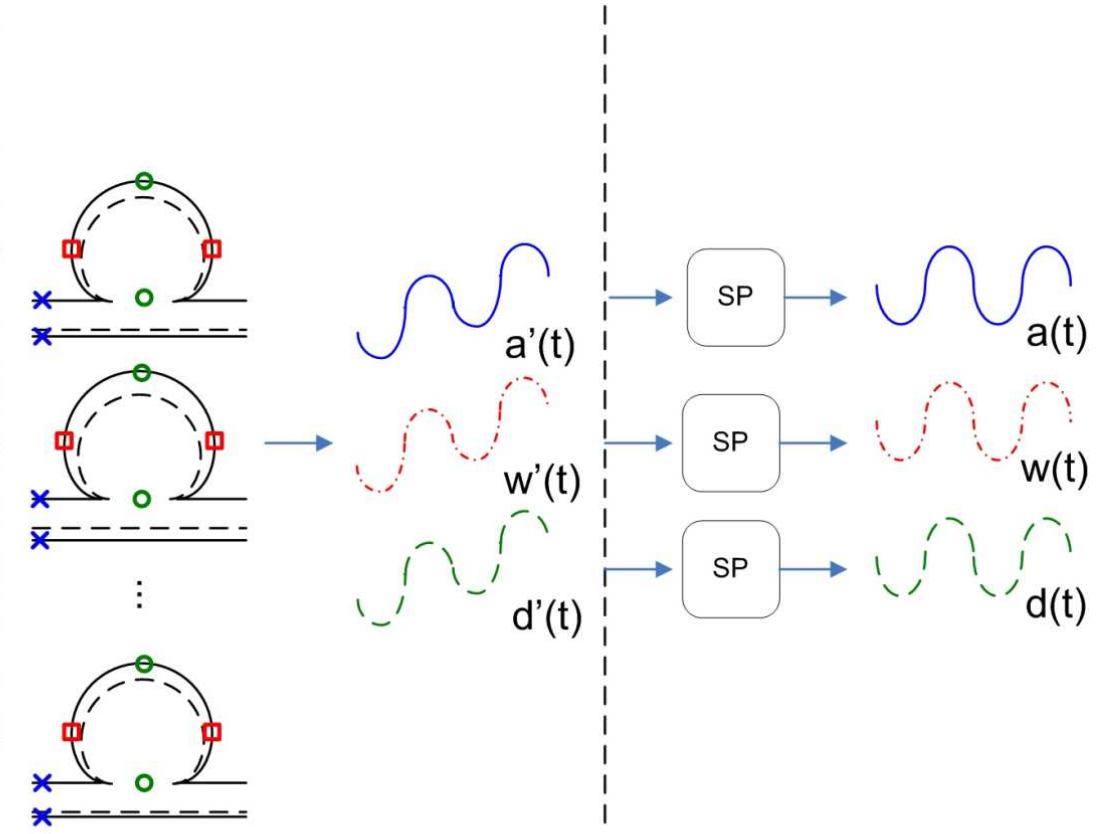

(2)

(3)

Figure 2. Wall motion estimation method.(1) image registration, (2) point propagation and feature quantification, and 3) post-processing. Crosses $(\times)$, circles $(\circ)$, and squares $(\square)$, show the points used to measure changes in the artery $(a(t))$, depth $(d(t))$, and width $(w(t))$ of the aneurysm. IR = image registration; $\mathrm{SP}=$ signal processing.

of the differential pulsation between the aneurysm and the artery, as explained later. For the spacing between control points employed in this paper, the spatial support of each B-Spline was in the order of the aneurysm size, and landmarks placed close to the aneurysm could be modified even in the absence of artery deformation. The points used to define the depth and with of the aneurysm were placed as shown in Figure 2.

2.2.3. Post-processing analysis. If changes in image intensity during the contrast filling were modeled linearly as $I_{t}(\mathbf{x})=\alpha I_{0}(\mathbf{x})+c$, with $\alpha$ and $c$ constant values, the use of MI-based IR would account for these changes in intensity. This is because MI-based IR is robust to changes in intensity scaleł as shown in Figure 3. However, intensity changes that occur during the contrast injection cannot be described accurately by this model, probably because of complex blood filling patterns. We have not found in the literature models of intensity changes due to contrast agent distribution to assess their influence on the IR, but a visual inspection of intensity profiles over time reveals that an exponential dependency of time is a reasonable approximation. Figure 4 shows that, even when the size of the aneurysm does not change, the optimizer will try to match the intensity profiles by scaling the moving image. This overestimation of the aneurysmal size results $\ddagger$ Note that this does not mean invariance of $M I$, in fact $M I(A, \alpha A)=H(A)+\log \|\alpha\|[26]$ 


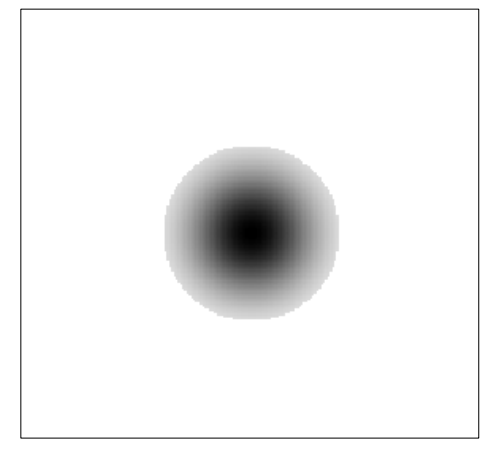

(a)

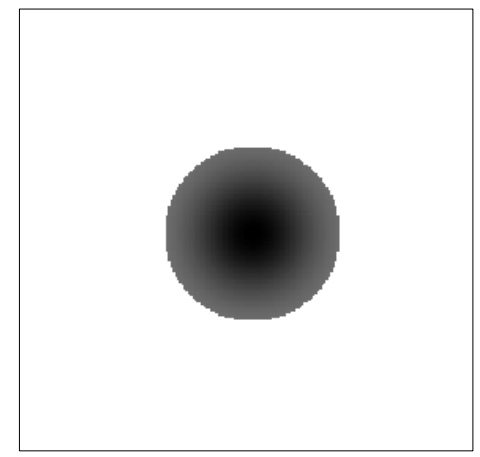

(b)

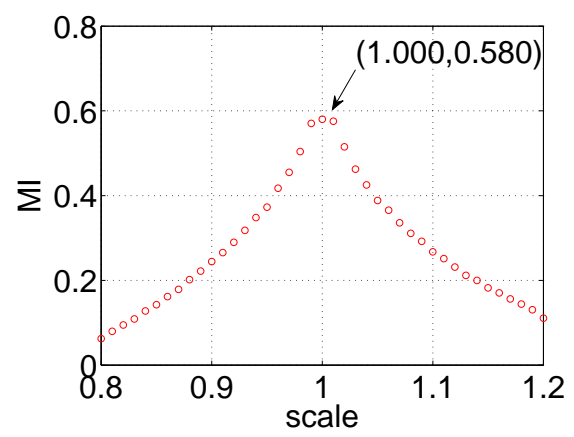

(c)

Figure 3. Robustness of MI to intensity scaling. In this example, the pixel intensity of the fixed image $I_{f}(\mathbf{x})$ (a) is half the intensity of moving image $I_{m}(\mathbf{x})(\mathrm{b})\left(I_{m}(\mathbf{x})\right.$ $\left.=0.5^{*} I_{f}(\mathbf{x})\right)$. (c) $M I\left(I_{f}, T_{i s}\left(I_{m}\right)\right)$ as a function of the scaling factor for an isotropic scaling transformation $T_{i s}$. MI has a maximum at 1 .

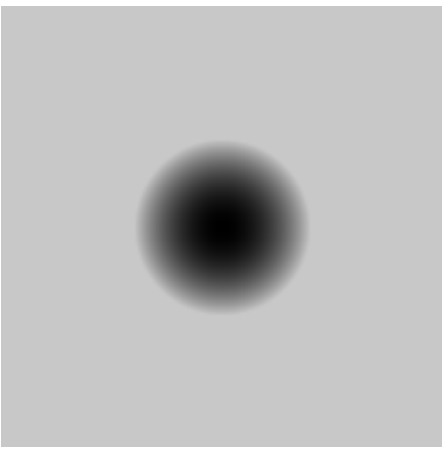

(a)

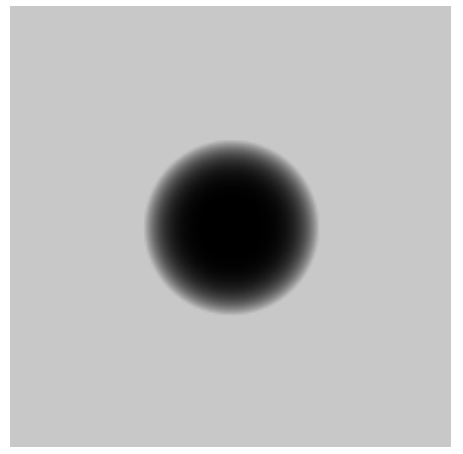

(b)

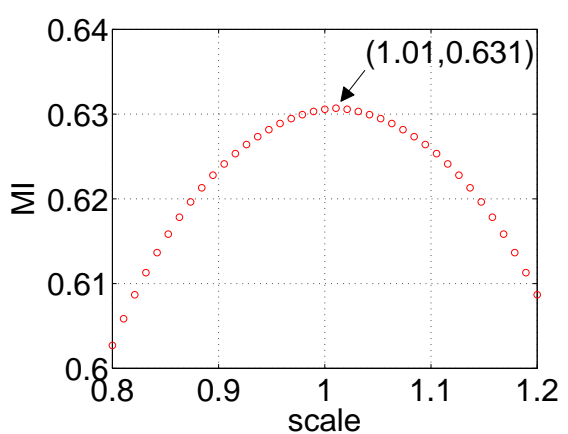

(c)

Figure 4. Overestimation of dilation when considering a non-linear relationship of intensities. In this example, the fixed image $I_{f}(\mathbf{x})$ (a) presents a quadratic intensity profile, whereas the moving Image $I_{m}(\mathbf{x})$ (b) presents a quartic profile. (c) $M I\left(I_{f}, T_{i s}\left(I_{m}\right)\right)$ as a function of the scaling factor for an isotropic scaling transformation $T_{i s}$. MI has a maximum at a value higher than 1 .

in an increase in the mean value of the dilation curves over time. Fortunately, this change has low frequency with respect to pulsation, and can be removed by applying high-pass filters (Figure 5).

\subsection{Differential pulsation index}

As mentioned in the introduction, measuring pulsation is important to investigate the potential connection between regional differences in pulsation and rupture status. This is supported by the concensus in the literature about a weakness of the vessel wall induced by a deficit in collagen and elastine $[27,28,29,30]$, components of the arterial wall playing an important role in its biomechanical properties [31]. What is more, collagenase and elastase activities seem to be increased in ruptured cerebral aneurysms versus unruptured aneurysms [27]. Assuming that this deficit is higher in the aneurysm 


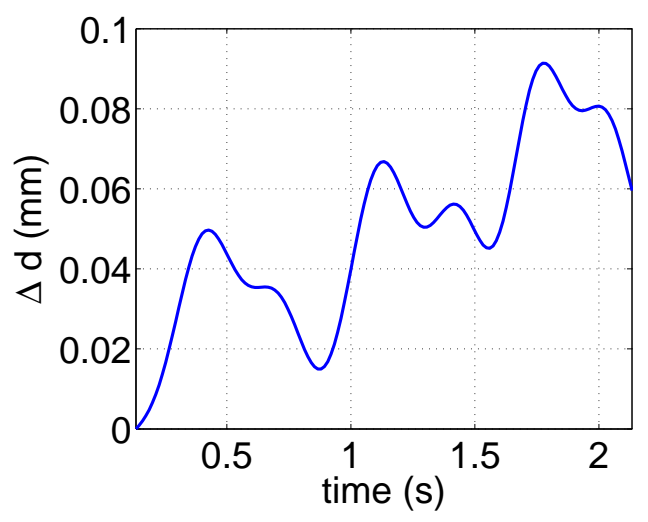

(a)

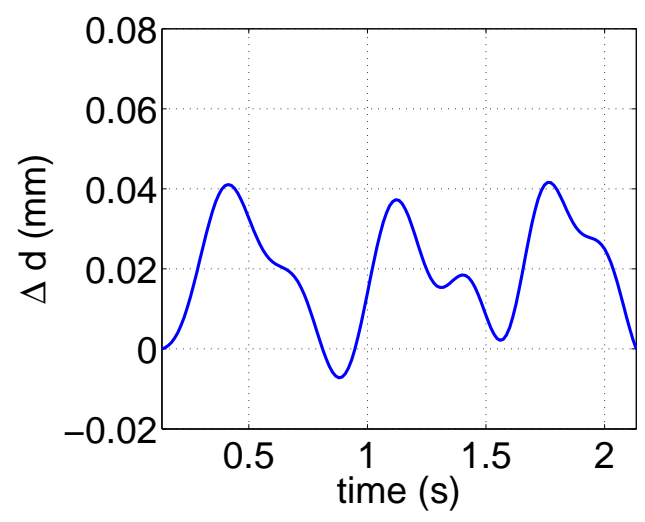

(b)

Figure 5. Distension waveform before (a) and after (b) filtering.

than in the parent artery, a difference in pulsation should be observed. To quantify this differential pulsation, we have defined the following index:

$$
\mu=\frac{|\max \{\hat{D}, \hat{W}\}-\hat{A}|}{\delta_{\text {art }}}
$$

where $\hat{D}, \hat{W}, \hat{A}$ represent the peak-to-peak amplitude of $d(t), w(t), a(t)$ respectively, and $\delta_{\text {art }}$ is the artery diameter. According to the definition, the index $\mu$ measures the difference in pulsation between aneurysm and artery, and expresses it as a fraction of the artery diameter. This normalization was added to compare aneurysms at different locations, since the same absolute difference in pulsation has a different meaning for arteries of different size. For example, a difference of $0.1 \mathrm{~mm}$ in pulsation is more important at the level of the ACA with respect to the ICA for purposes of correlation with rupture.

\section{Results}

\subsection{Accuracy}

To establish whether the detected motion could be discriminated from the intra-observer variability in delineating contours, a manual segmentation was performed by an expert observer in the first frame of patient \#7 by selecting closely located points on the boundary of the aneurysm and subsequently fitting a spline to the series of points. By considering also the independently selected landmarks for this same frame, the distributions of distances derived from: a) original landmarks to the spline, and b) propagated landmarks and original landmarks, were compared. To this end, Student paired t-test and ranked sum Wilcoxon tests were performed. As established before, the landmarks were tracked in the complete series in order to quantify the wall motion. The sequence \#7 presents features that make it suitable for this analysis like high signal-to-noise ratio, clear definition of borders, and visual pulsation. Figure 6 


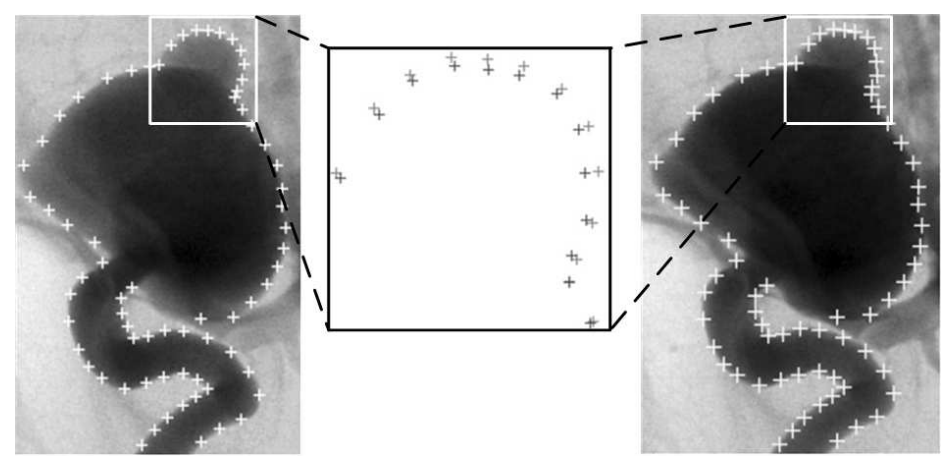

Figure 6. Illustration of the propagation of the landmarks between different frames. [left] patient \#7-frame \#1, [middle] zoom corresponding to region containing the lobule, [right] patient \#7-frame \#2.

illustrates the landmarks propagation from which wall deformation estimates follow. Statistically significant differences were found between the two distributions for 8/11 frames $\left(p_{T T E S T}<0.04, p_{W I L}<0.02\right)$. In all of these cases, the average distance between propagated landmarks and original landmarks was larger than that due to intra-observer variability. Thus, although small deformation fields are obtained for all the images within the temporal series (see Figure 6), these differ in a statistically meaningful way from the error made in the manual delineation of the contours.

\subsection{Distension curves}

Figure 7 shows $d(t), w(t)$, and $a(t)$ for sequences exhibiting wall motion. To distinguish curves carrying information about wall motion from those containing just measurement noise, we have compared them to the model in Figure 10, and the presence/absence of wall motion was assessed based on the similarity. Since contrast injection times depend on the specific protocols of each clinical center, we show deformations for a single cardiac cycle for normalization purposes. As a consequence of the image acquisition issues mentioned Section 4, curves present distortions in some time intervals. This occurs in particular at the beginning and at the end of the contrast injection, where the largest changes in the image take place. Therefore, when information was available for several cardiac cycles, we selected the central part of the acquisition window to minimize distortions. Table 3 presents parameters extracted from $d(t), w(t)$, and $a(t)$ for all sequences in Figure 7.

\subsection{Differential pulsation and rupture status}

Figure 8 (a) shows the distribution of the differential pulsation $\mu$ defined in Section 2.3 with respect to the rupture status. As shown in the Figure, the distribution of $\mu$ for ruptured aneurysms presents a higher mean value than the distribution for unruptured aneurysms $\left(\bar{\mu}_{R}=6.1 \%\right.$ vs. $\left.\bar{\mu}_{U}=1.3 \%\right)$. As the standard deviations are not small, a two-tailed unpaired $t$-test was performed to assess the statistical significance of these 


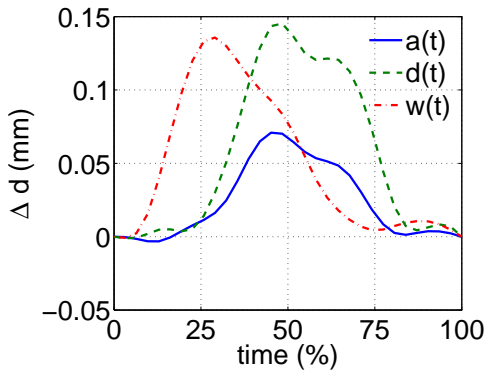

\#1

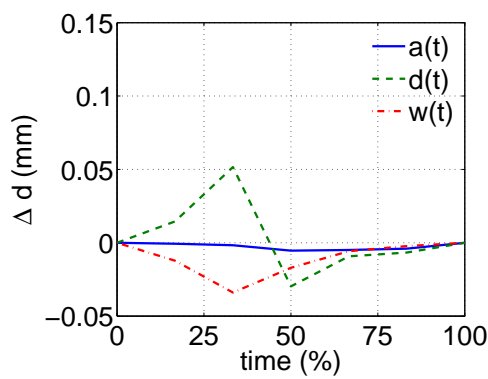

\#6

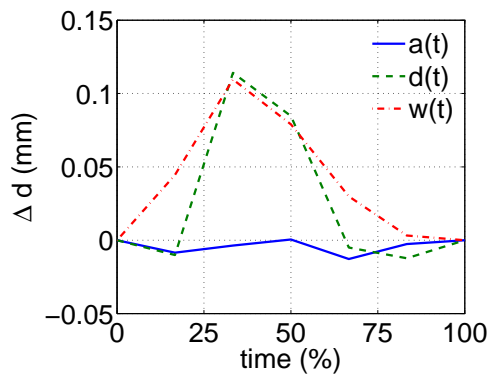

\#9

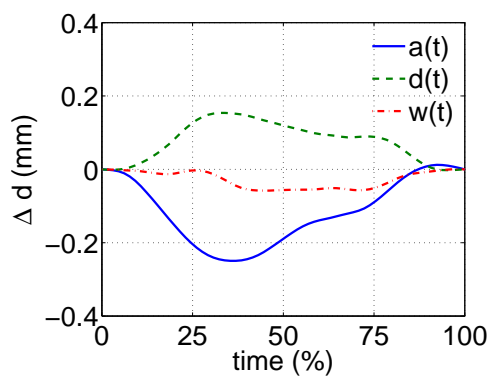

\#15

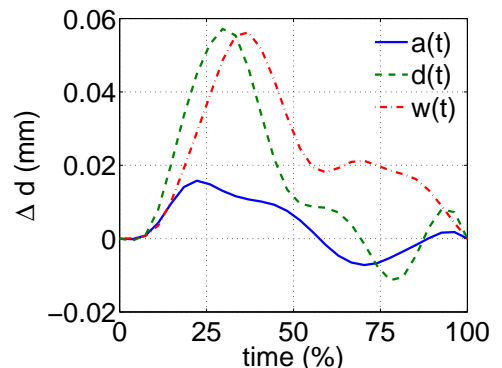

\#2

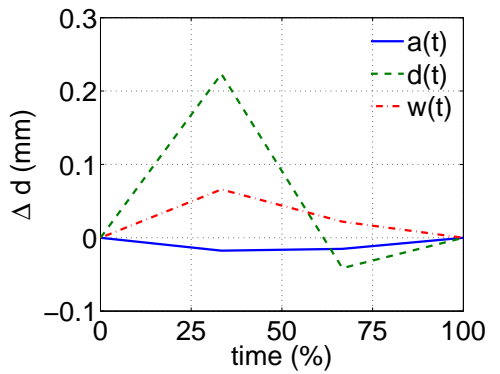

\#7

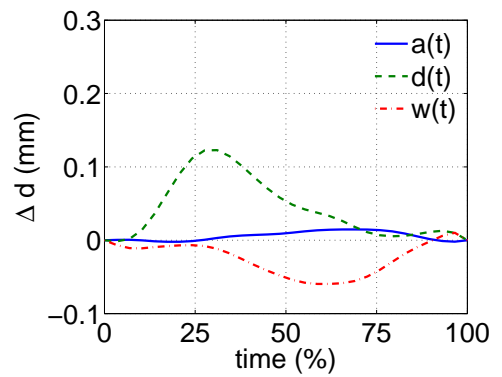

\#10

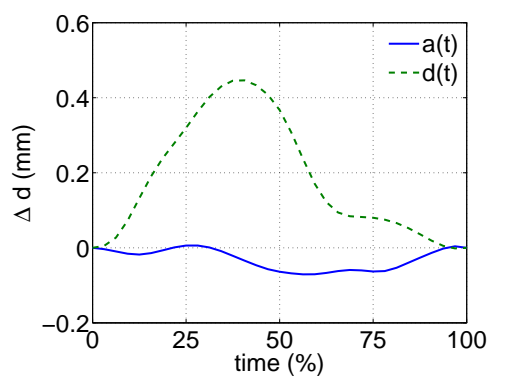

\#16

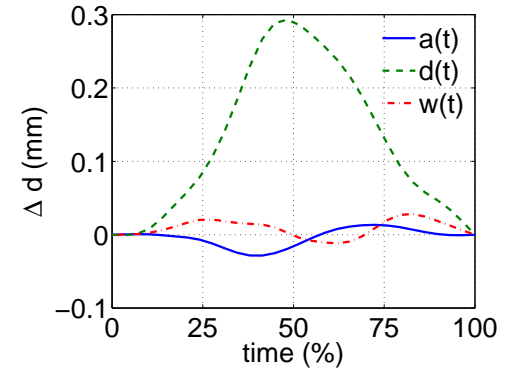

\#3

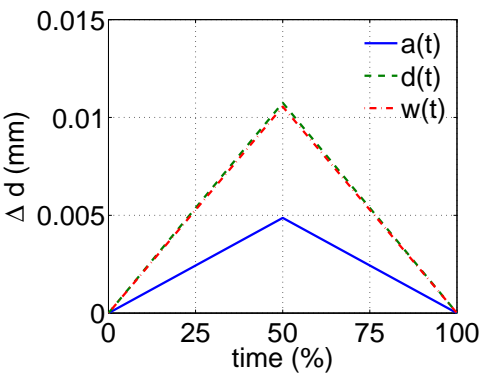

\#8

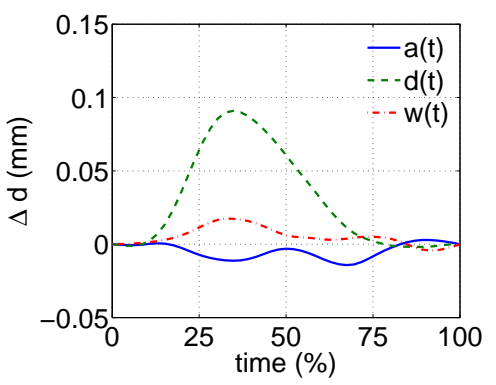

\#12

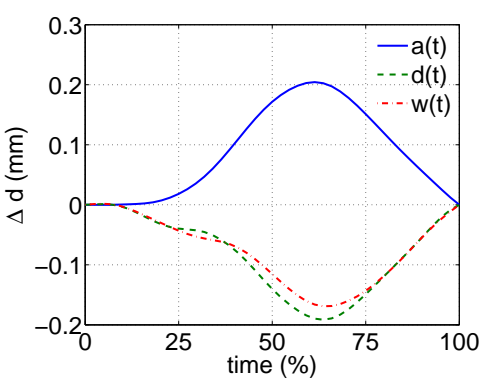

\#18

Figure 7. Distention curves $a(t), d(t)$, and $w(t)$ over the cardiac cycle for sequences showing wall motion. Time is expressed as percent of the cardiac cycle. $w(t)$ is omitted for sequence \#16, since it could not be estimated because of defects in contrast filling. 
Table 3. Parameters extracted from curves $a(t), d(t)$, and $w(t)$ for sequences showing wall motion. $\mathrm{R}=$ Ruptured, $\mathrm{U}=$ Unruptured; $\mu$ = differential pulsation; $\hat{D}, \hat{W}, \hat{A}=$ peak-to-peak amplitude of $d(t), w(t), a(t)$ respectively; $\phi_{d a}, \phi_{d w}, \phi_{w a}=$ phase difference $d(t)-a(t), d(t)-w(t)$, and $w(t)-a(t)$, expressed as percent of the cardiac cycle.

\begin{tabular}{lllllllll}
\hline $\begin{array}{l}\text { Patient } \\
\#\end{array}$ & $\begin{array}{l}\text { Status } \\
\mathrm{R} / \mathrm{U}\end{array}$ & $\begin{array}{l}\boldsymbol{\mu} \\
\%\end{array}$ & $\begin{array}{l}\hat{\mathbf{D}} \\
m m\end{array}$ & $\begin{array}{l}\hat{\mathbf{W}} \\
m m\end{array}$ & $\begin{array}{l}\hat{\mathbf{A}} \\
m m\end{array}$ & $\begin{array}{l}\phi_{\text {da }} \\
\%\end{array}$ & $\begin{array}{l}\phi_{\text {wa }} \\
\%\end{array}$ & $\begin{array}{l}\phi_{\text {dw }} \\
\%\end{array}$ \\
\hline 1 & $\mathrm{U}$ & 2.26 & 0.15 & 0.14 & 0.074 & 0 & -16 & 20 \\
2 & $\mathrm{U}$ & 2.40 & 0.07 & 0.06 & 0.023 & 7 & 13 & -7 \\
3 & $\mathrm{R}$ & 15.97 & 0.29 & 0.04 & 0.042 & -25 & 6 & -34 \\
6 & $\mathrm{R}$ & 5.16 & 0.08 & 0.03 & 0.005 & 33 & 0 & 33 \\
7 & $\mathrm{R}$ & 8.02 & 0.26 & 0.07 & 0.018 & 31 & 31 & 0 \\
8 & $\mathrm{U}$ & 0.17 & 0.01 & 0.01 & 0.005 & 0 & 0 & 0 \\
9 & $\mathrm{R}$ & 3.53 & 0.14 & 0.11 & 0.013 & -16 & -16 & 0 \\
10 & $\mathrm{U}$ & 2.34 & 0.12 & 0.07 & 0.017 & -47 & 13 & 28 \\
12 & $\mathrm{R}$ & 3.71 & 0.09 & 0.02 & 0.017 & 46 & 46 & 0 \\
15 & $\mathrm{U}$ & 2.84 & 0.16 & 0.06 & 0.262 & 40 & 9 & 31 \\
$16^{\mathrm{a}}$ & $\mathrm{U}$ & 8.25 & 0.45 & - & 0.077 & 39 & - & - \\
18 & $\mathrm{U}$ & 0.52 & 0.19 & 0.17 & 0.204 & 38 & 38 & 0 \\
\hline
\end{tabular}

a It was not possible to estimate $w(t)$ for patient \#16 because of defects in the contrast filling. Therefore, measurements involving this curve are omitted in this table.

Table 4. Correlation values between $\mu, \Delta d_{\text {max }}$, and $\log (v o l$.) according to the rupture status. $p$-values are shown between brackets, in bold when the correlation is considered significant $(p<0.05)$.

\begin{tabular}{llll}
\hline Magnitude & Ruptured & Unruptured & All \\
\hline$\mu$ & $0.55(0.25)$ & $0.67(\mathbf{0 . 0 2})$ & $0.53(\mathbf{0 . 0 3})$ \\
$\Delta d_{\max }$ & $0.76(0.08)$ & $0.56(0.07)$ & $0.61(\mathbf{0 . 0 1})$ \\
\hline
\end{tabular}

differences. The result of such test showed that the null hypothesis of equal means is rejected for $p=0.05$, i.e. the differences are significant at a level of $5 \%$.

\subsection{Variations according to type}

We have also investigated variations in the pulsation magnitude according to type and size. Figure 8 (b) shows that terminal aneurysms present a higher pulsation amplitude than lateral aneurysms, but this difference is not significant according to a $t$-test. Figure 9 shows a linear correlation between $\mu, \Delta d_{\text {max }}$, and the logarithm of the aneurysm volume $\log (\mathrm{vol}$.$) . Table 4$ summarizes the correlation values between the magnitudes. These results are in agreement with the difference in mean volume between ruptured $\left(\bar{v}_{R}=0.91 \mathrm{~cm}^{3}\right)$ and unruptured $\left(\bar{v}_{U}=0.58 \mathrm{~cm}^{3}\right)$ aneurysms. 


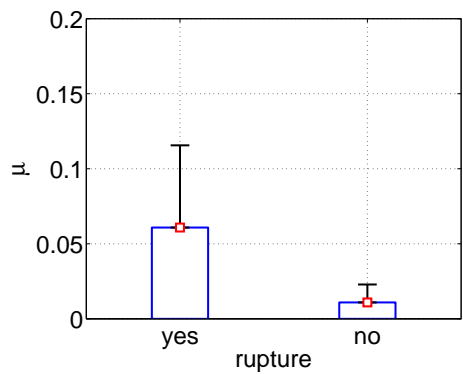

(a)

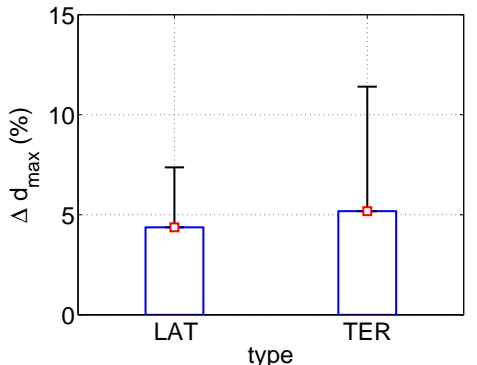

(b)

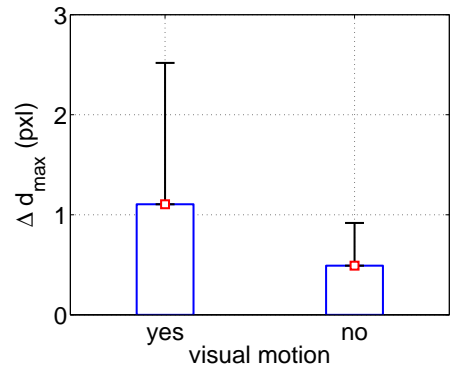

(c)

Figure 8. Pulsation of aneurysms according to (a) rupture status, (b) type, and (c) visual motion. Note that the $y$ axes units are different for all figures: the $\mu$ index is plotted in (a), the maximal deformation (relative to the artery diameter) in (b), and image pixels in (c). Bars show the standard deviations of distributions. The $\mu$ value for sequence \#16 was considered as an outlier, and excluded in (a).

\subsection{Measured vs. observed pulsation}

To study the consistency between measured and observed pulsation, the presence of visual pulsation was assessed by two observers in two sessions separated by one week. According to the Kappa statistics, the intra-observer accuracy is 0.76 for both observers (substantial agreement according to Landis and Koch's paper [32]) and the interobserver accuracy of 0.41 (moderate agreement). The reason why a perfect agreement was not achieved is most likely due to the fact that the wall motion amplitudes are quite small, and in many cases close to the image resolution. Figure 8 (c) shows the distribution of pulsation amplitudes according to presence of visual motion as assessed by the observers mentioned in Section 4. As expected, aneurysms classified as "pulsating" presented a higher pulsation amplitude in mean than "non-pulsating" aneurysms.

\section{Discussion}

In previous work $[5,15]$, we described temporal changes in aneurysms by measuring the displacements of points on the vessel wall. This approach requires a previous segmentation to assess the sign of the displacement, i.e. whether points move inwards or outwards the aneurysm at the reference point. Besides adding an extra step in the pipeline, (according to our experience) the segmentation of DSA images is extremely difficult since this modality does not provide a proper definition of the vessel lumen. As the wall displacement is in the order of the image resolution, even small segmentation errors can make the method fail to recover pulsation. In this paper we have circumvented this drawback by measuring the euclidean distance between pairs of points, which avoids the need for a previous image segmentation.

In some cases, we found a rigid motion associated to the global pulsation of the intracranial vasculature that should be corrected before estimating the deformation. 


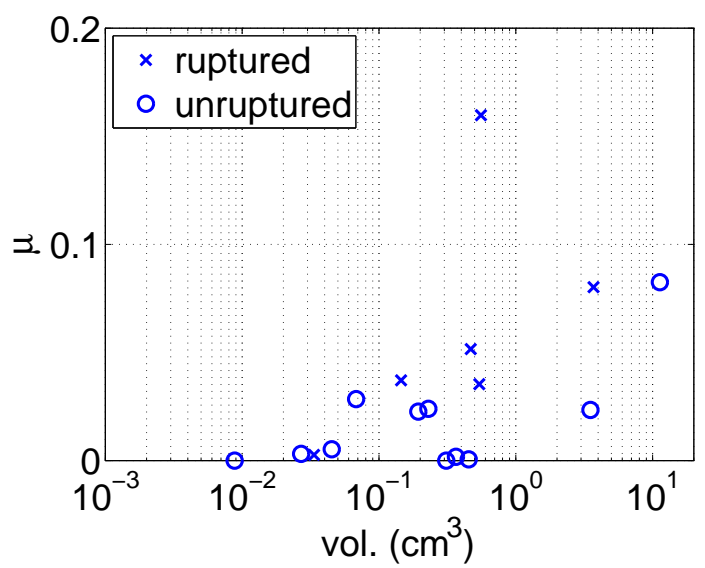

(a)

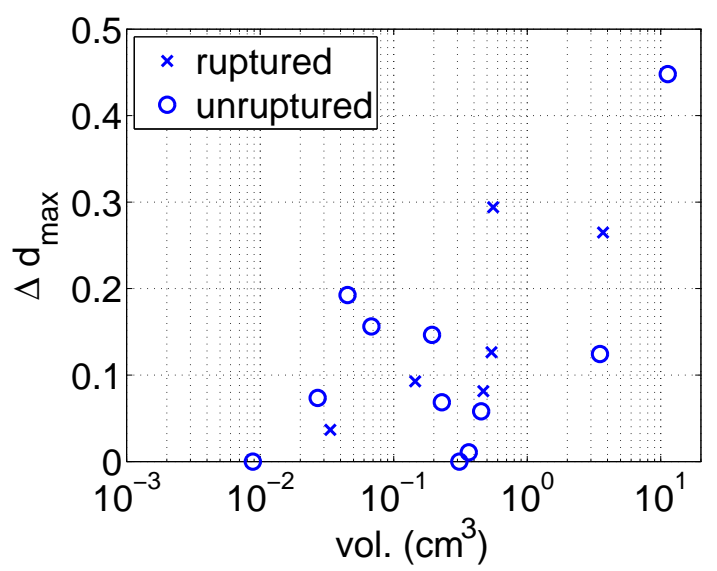

(b)

Figure 9. Variations in the magnitude of pulsation with the size of the aneurysm. (a) $\mu$ vs volume, (b) Maximum displacement vs. volume. The size of the aneurysms was measured as the volume of the prolate ellipsoid with minor radii $a=b=$ with and major radius $c=$ depth. The volume is represented by using a logarithmic scale, since this is more appropriate for the distribution of volumes in our database.

Fortunately, this motion was very small $\S$ and was captured by the non-rigid transformation. Therefore, the rigid motion was corrected automatically without adding an extra step.

It is important to make some remarks with respect to image acquisition. The Nyquist theorem establishes that the minimum sampling frequency $f_{s}^{\text {min }}$ that allows recovering the aneurysmal pulsation is twice the signal bandwidth (BW) [33]. To our knowledge, there are no measurements of temporal changes of intracranial aneurysms in the literature to estimate BW, and we have used simulated pressure data [34] for the Common Carotid Artery (CCA) (Figure 10) to estimate it. Two important assumptions are made here. 1) The wall displacement and pressure waveforms are the same (except for a scale factor): this is true if linear elasticity of the vessel wall is assumed, but both waveforms could differ slightly if a viscoelastic model and large mass-effects are considered. 2) The pressure waveform does not change from the CCA to the location of the aneurysm. Figure 10 shows that the most important frequency components are comprised in the range $0-4 \mathrm{~Hz}$, and therefore we can establish a $f_{s}^{\min }=8 \mathrm{~Hz}$ as the minimum $f_{s}$ to recover aneurysmal pulsation. Even when this estimation is based on simulated data, it allowed obtaining curves resembling the typical arterial pressure waveform [35] (see for example sequences \#1, \#2, \#9, \#10, \#12, \#15 in Figure 7 ). This similarity supports the assumption that the recovered deformations effectively quantify the wall displacements of the vessel, and do not come from sources of variations like image noise, intensity changes, or movement artifacts.

As shown in Table 2, some sequences were acquired at lower sampling frequency $\S$ Note that the aneurysmal pulsation is also very small, and therefore any rigid motion must be completely compensated to have an accurate estimation 


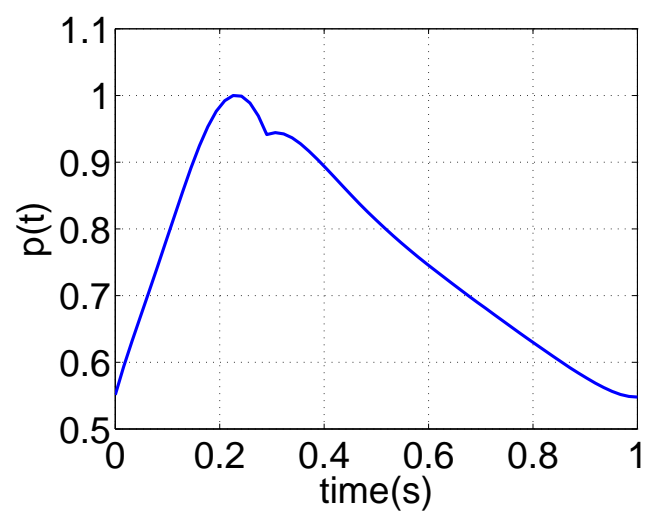

(a)

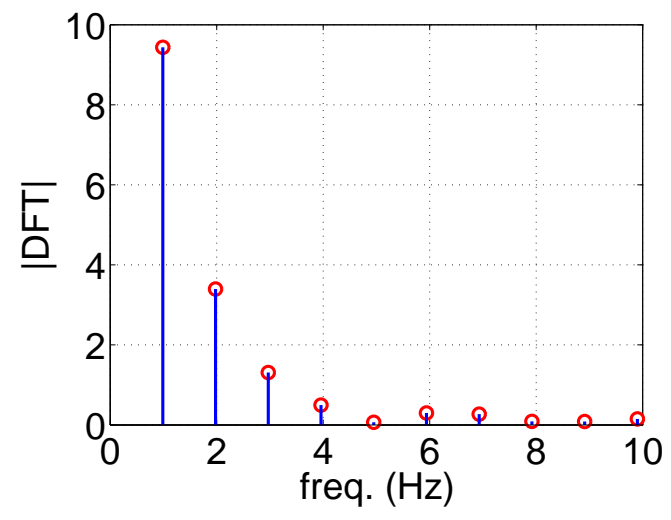

(b)

Figure 10. (a) Simulated pressure at the Common Carotid Artery (CCA) [34] (b) Magnitude of its DFT (spacing of samples in the Fourier domain $1 / T=1$, where $T$ is the period of the signal). The continuous component $(f=0)$ was removed from the DFT for better visualization of high frequency components. Frequencies higher than $20 \mathrm{~Hz}$ were also omitted because of their small amplitude.

than this inferior limit. Sometimes, low $f_{s}$ values are preferred to obtain a higher image quality, and values as low as $2 \mathrm{~Hz}$ can be found. Figure 7 shows the result when $f_{s}$ does not meet the requirements imposed by the Nyquist's theorem (sequences \#7 and \#8). As the sampling frequency for these sequences $\left(f_{s}=3 \mathrm{~Hz}\right.$ and $f_{s}=2 \mathrm{~Hz}$, respectively), distension waveforms cannot be completely recovered. This has an influence on the correlation with rupture, since three of these sequences belong to the group of ruptured aneurysms. However, the fact of missing a peak has as a consequence a reduction in the differences between ruptured and unruptured aneurysms (already found significant). If the peak were not missed the differences between groups would be even larger.

The dependency of the method on the contrast injection parameters (e.g. injection rate, total volume) is critical, since they must ensure a complete and homogeneous filling of the aneurysm for at least one cardiac cycle. It is very difficult to select a fixed set of parameters to satisfy these requirements in all aneurysms, since the filling depends strongly on factors like size, shape, and type of the aneurysm. The influence of type is well illustrated by terminal aneurysms of the basilar artery (Figure 1, patients $\# 3$ and \#6). In these cases, the aneurysm receives the blood jet directly from the parent artery which produces a strong washout of contrast medium. A large size may be also problematic, since the filling is slower and sometimes the injected contrast is not sufficient to fill the aneurysm (Figure 1, patients \#7 and \#10). These problems are related to the upper limits imposed by the cellular effects of contrast media [36], and their connection to nephropathies [37]. If there were no such limits, the amount of contrast could be made big enough to compensate the effects of size and type.

Figure 7 shows that in some cases the recovered curves are out-of-phase. This cannot be attributed to the filters used for postprocessing, since the phase difference 


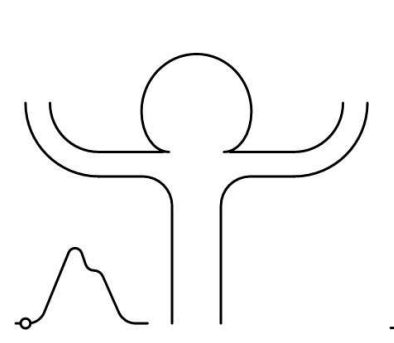

(a)

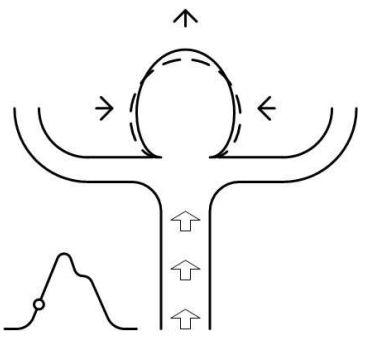

(b)

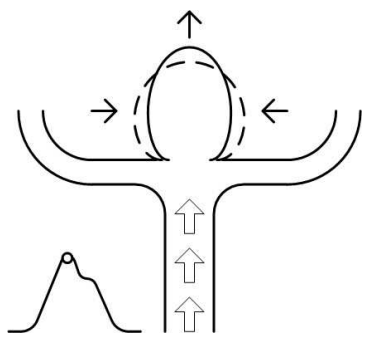

(c)

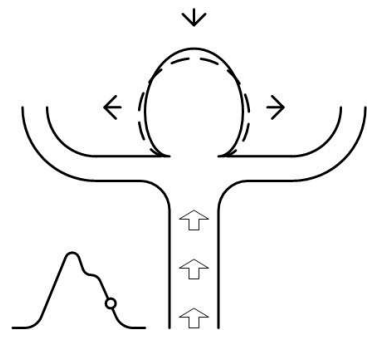

(d)

Figure 11. Relationship between blood flow and wall motion for a basilar aneurysm. Figures (a)-(d) show the deformation of the aneurysm for four temporal points of the cardiac cycle (shown as an empty circle at bottom left of each figure). Empty arrows in the parent artery represent the blood blow (their length is proportional to the flow magnitude). The small black arrows represent the wall displacement with respect to the previous time instant.

persists when filters are not applied (see patient \# 6 for example). A possible explanation to this phenomenon is the asymmetric deformation of the aneurysm owing to specific blood flow patterns and asymmetric weakness of the arterial wall. Consider for example the same patient (\#6). As can be seen from Figure 1 this is a basilar aneurysm that receives a direct impingement of the blood flow coming from the basilar artery, which produces a differential pressure between the impingement region and the rest of the aneurysm. On the other hand, Meng et al. [38] have performed studies showing localized aneurysmal-type remodeling in regions of accelerating flow (in our case the region of impingement), which could create asymmetric weakness of the arterial wall. The combined effect of differential pressure and asymmetric weakness could explain the increase in depth at the expense of a decrease in width as shown in Figure 11. This explanation predicts a phase difference of 180 degrees, which in fact was the difference of phase found in the example. A similar analysis of other aneurysmal configurations should be performed to understand the regional differences in pulsation, and a CFD analysis could be useful for understanding the connection with the haemodynamics. Another explanation for these phase differences is the pulsation of extravascular structures (like the brain), which could pulsate synchronously with the artery and modify influence the expected deformation pattern of the aneurysm.

Lateral and terminal aneurysms showed slight non significant differences in wall motion. However, terminal aneurysms receive a direct impact of the blood jet coming from the parent artery which should produce a larger deformations. A possible explanation are the differences in location of the considered aneurysms, which should be normalized to ensure similar haemodynamic conditions. Larger aneurysms exhibited a higher differential pulsation index, and wall motion amplitude. However, other papers have reported no relationship between aneurysm size and volume increases [2].

The method presented in this paper allows obtaining deformations in the image plane. This information could be complemented by a second acquisition in an orthogonal projection. However, according to our experience, the neighboring vessels make 


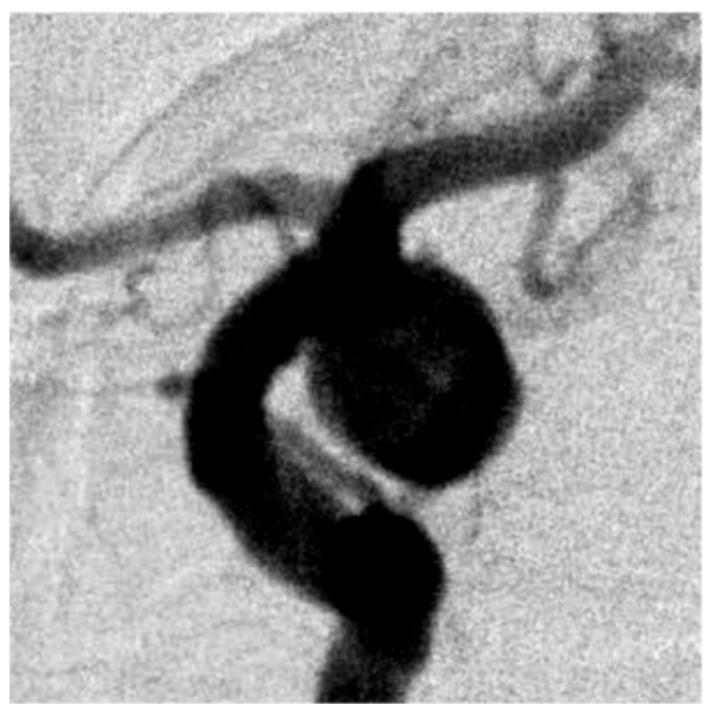

(a)

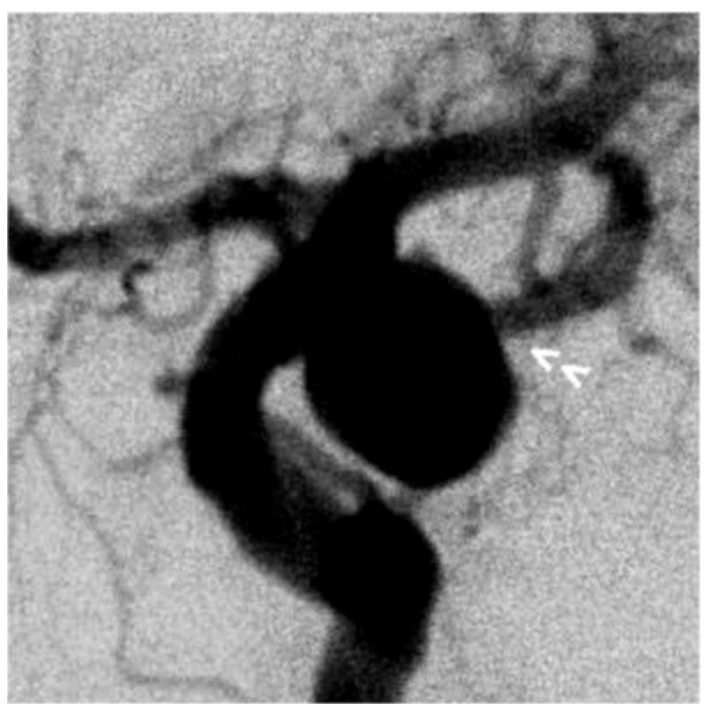

(b)

Figure 12. Influence of neighboring vessels: at the beginning of the contrast filling the boundary of the aneurysm is free (a). As the contrast injection progresses, distal vessels start appearing in the image causing local misregistration occurs due to lack of point correspondence.

extremely difficult to obtain two orthogonal views showing the aneurysm without an overlapping with other vessels. The presence of neighboring vessels can also perturb the recovered displacement fields: at the beginning of the contrast filling, the aneurysm appears isolated from the rest of the vasculature and its boundary is well defined. As the contrast injection progresses, distal vessels start appearing in the image (Figure 12) and a local misregistration occurs due to the lack of correspondence [39].

In this paper we have found statistically significant differences in the index $\mu$ according to the rupture status. However, even when there are some follow-up studies of unruptured aneurysms [40], the criterion adopted by the clinical centers involved in our study is to treat the aneurysm (coiling or stenting) immediately after identification by angiography to avoid the risk of hemorrhage. Therefore, the available sequences do not allow studying differences in pulsation before and after rupture [2, 41]. This is a limitation of most of works on rupture found in the literature. Therefore the underlying hypothesis of the experiments presented in this paper is that the pulsation properties of the aneurysm immediately before rupture are similar to those of ruptured aneurysms. 


\section{Conclusions}

In this paper we have combined non-rigid registration methods with signal processing techniques to quantify wall motion of intracranial aneurysms from dynamic DSA sequences. We applied the presented methodology to a series of intracranial aneurysms and found a higher index of differential pulsation for ruptured aneurysms. These measurements and observations may help us better stratify aneurysm rupture risk and understand the wall motion effects on aneurysm haemodynamics and their evolution.

\section{References}

[1] D. O. Wiebers, J. P. Whisnant, J. Huston III, I. Meissner, R. D. Brown Jr., D. G. Piepgras, G. S. Forbes, K. Thielen, D. Nichols, W. M. O'Fallon, J. Peacock, L. Jaeger, N. F. Kassell, G. L. Kongable-Beckman, and J. C. Torner. International study of unruptured intracranial aneurysms investigators. unruptured intracranial aneurysms: Natural history, clinical outcome, and risks of surgical and endovascular treatment. Lancet, 362(9378):103-10, July 2003.

[2] F. B. Meyer, J. Huston III, and S. S. Riederer. Pulsatile increases in aneurysm size determined by cine phase-contrast MR angiography. Journal of Neurosurgery, 78(6):879-83, June 1993.

[3] J. Ueno, T. Matsuo, K. Sugiyama, and R. Okeda. Mechanism underlying the prevention of aneurismal rupture by coil embolization. Journal of Medical and Dental Sciences, 49(4):135-41, December 2002.

[4] Y. Kato, M. Hayakawa, H. Sano, M. V. Sunil, S. Imizu, M. Yoneda, S. Watanabe, M. Abe, and T. Kanno. Prediction of impending rupture in aneurysms using 4D-CTA: histopathological verification of a real-time minimally invasive tool in unruptured aneurysms. Minimally Invasive Neurosurgery, 47(3):131-5, June 2004.

[5] L. Dempere, E. Oubel, M. A. Castro, C. M. Putman, A. F. Frangi, and J. R. Cebral. CFD analysis incorporating the influence of wall motion: Application to intracranial aneurysms. In R. Larsen, M. Nielsen, and J. Sporring, editors, 8th International Conference on Medical Image Computing and Computer-Assisted Intervention, MICCAI 2006, volume 4190 of Lecture Notes in Computer Science, pages 438-45, Copenhagen, Denmark, October 2006. Springer.

[6] V. Yaghmai, M. Rohany, A. Shaibani, M. Huber, H. Soud, E. J. Russell, and M. T. Walker. Pulsatility imaging of saccular aneurysm model by 64 -slice CT with dynamic multiscan technique. Journal of Vascular and Interventional Radiology, 18(6):785-8, June 2007.

[7] H. G. Boecher-Schwarz, K. Ringel, L. Kopacz, A. Heimann, and O. Kempski. Ex vivo study of the physical effect of coils on pressure and flow dynamics in experimental aneurysms. American Journal of Neuroradiology, 21(8):1532-6, September 2000.

[8] C. Zhang, M. C. Villa-Uriol, M. De Craene, J. M. Pozo, and A. F. Frangi. Time-resolved 3D rotational angiography reconstruction: towards cerebral aneurysm pulsatile analysis. In H. U. Lemke, K. Inamura, K. Doi, M. W. Vannier, and A. G. Farman, editors, International Journal of Computer Assisted Radiology and Surgery, volume 3, pages S44-S46, Barcelona, Spain, June 2008. Springer.

[9] J. M. Wardlaw, J. Cannon, P. F. Statham, and R. Price. Does the size of intracranial aneurysms change with intracranial pressure? Observations based on color power transcranial Doppler ultrasound. Journal of Neurosurgery, 88(5):846-50, May 1998.

[10] P. R. Hoskins, J. Prattis, and J. Wardlaw. A flow model of cerebral aneurysms for use with power Doppler studies. British Journal of Radiology, 71(841):76-80, January 1998.

[11] F. Ishida, H. Ogawa, T. Simizu, T. Kojima, and W. Taki. Visualizing the dynamics of cerebral aneurysms with four-dimensional computed tomographic angiography. Neurosurgery, 57(3):460$71,2005$. 
[12] M. Hayakawa, K. Katada, H. Anno, S. Imizu, J. Hayashi, K. Irie, M. Negoro, Y. Kato, T. Kanno, and H. Sano. CT angiography with electrocardiographically gated reconstruction for visualizing pulsation of intracranial aneurysms: Identification of aneurysmal protuberance presumably associated with wall thinning. American Journal of Neuroradiology, 26(6):1366-9, 2005.

[13] R. Manzke, M. Grass, T. Nielsen, G. Shechter, and D. Hawkes. Adaptive temporal resolution optimization in helical cardiac cone beam CT reconstruction. Medical Physics, 30(12):3072-80, December 2003.

[14] M. Matsumoto, T. Sasaki, K. Suzuki, J. Sakuma, Y. Endo, and K. Kodama. Visualizing the dynamics of cerebral aneurysms with four-dimensional computed tomographic angiography. Neurosurgery, 58(5):E1003, May 2006.

[15] E. Oubel, M. De Craene, C. M. Putman, J. R. Cebral, and A. F. Frangi. Analysis of intracranial aneurysm wall motion and its effects on hemodynamic patterns. In A. Manduca and X.P. Hu, editors, SPIE Medical Imaging 200\%: Physiology, Function, and Structure from Medical Images, volume 6511, pages 10-11, San Diego, CA, USA, February 2007. SPIE press.

[16] J. P. Thirion. Image matching as a diffusion process: an analogy with Maxwell's demons. Medical Image Analysis, 2(3):243-60, September 1998.

[17] J. Weickert, A. Bruhn, T. Brox, and N. Papenberg. Mathematical Models for Registration and Applications to Medical Imaging, volume 10 of Mathematics in Industry, chapter A Survey on Variational Optic Flow Methods for Small Displacements, pages 103-36. Springer, 2006.

[18] S. Balter, D. Ergun, E. Tscholl, F. Buchmann, and L. Verhoeven. Digital substraction angiography: Fundamental characteristics. Radiology, 152(1):195-8, July 1984.

[19] A. Collignon, F. Maes, D. Delaere, D. Vandermeulen, P. Suetens, and G. Marchal. Automated multi-modality image registration based on information theory. Lecture Notes in Computer Science, pages 263-74, Ile de Berder, France, June 1995. Kluwer Academic.

[20] P. Viola and W. M. Wells. Alignment by maximization of mutual information. In E. Grimson, S. Shafer, A. Blake, and K. Sugihara, editors, Fifth International Conference on Computer Vision, pages 16-23, Los Alamitos, CA, USA, June 1995.

[21] S. Lee, G. Wolberg, K-W. Chwa, and S. Y. Shin. Image metamorphosis with scattered feature constraints. IEEE Transactions on Visualization and Computer Graphics, 2(4):33754, December 1996.

[22] S. Lee, G. Wolberg, and S. Y. Shin. Scattered data interpolation with multilevel B-splines. IEEE Transactions on Visualization and Computer Graphics, 3(3):228-44, July-September 1997.

[23] D. Rueckert, L. I. Sonoda, C. Hayes, D. L. G. Hill, M. O. Leach, and D. J. Hawkes. Nonrigid registration using free-form deformations: Application to breast MR images. IEEE Transactions on Medical Imaging, 18(8):712-21, August 1999.

[24] F. L. Bookstein. Principal warps: Thin-plate splines and the decomposition of deformations. IEEE Transactions on Pattern Analysis and Machine Intelligence, 11(6):567-585, June 1989.

[25] M. H. Davis, A. Khotanzad, D. P. Flamig, and S. E. Harms. A physics-based coordinate transformation for 3-D image matching. 16(3):317-328, 1997.

[26] T. M. Cover and J. A. Thomas. Elements of information theory. Wiley-Interscience, 1991.

[27] P. Gaetani, F. Tartara, V. Grazioli, F. Tancioni, L. Infuso, and R. Rodriguez y Baena. Collagen cross-linkage, elastolytic and collagenolytic activities in cerebral aneurysms: a preliminary investigation. Life Sciences, 63(4):285-92, June 1998.

[28] B. J. Rajesh, S. Sandhyamani, and R. N. Bhattacharya. Clinico-pathological study of cerebral aneurysms. Neurology India, 52(1):82-6, March 2004.

[29] A. I. Holodny, M. Deck, and C. K. Petito. Induction and subsequent rupture of aneurysms of the circle of willis after radiation therapy in Ehlers-Danlos syndrome: a plausible hypothesis. American Journal of Neuroradiology, 17(2):226-32, February 1996.

[30] T. Mizutani and H. Kojima. Clinicopathological features of non-atherosclerotic cerebral arterial trunk aneurysms. Neuropathology, 20(1):91-7, March 2000.

[31] E. Fonck, G. Prod'hom, S. Roy, L. Augsburger, D. A. Rüfenacht, and N. Stergiopulos. Effect of 
elastin degradation on carotid wall mechanics as assessed by a constituent-based biomechanical model. American journal of physiology. Heart and circulatory physiology, 292(6):H2754-63, January 2007.

[32] J. R. Landis and G. G. Koch. The measurement of observer agreement for cathegorical data. Biometrics, 33(1):159-74, March 1977.

[33] A. V. Oppenheim and R. W Schafer. Digital Signal Processing. Prentice Hall, 1999.

[34] J. Alastruey, K. Parker, J. Peiró, S. Byrd, and S. Sherwin. Modelling the circle of Willis to assess the effects of anatomical variations and occlusions on cerebral flows. Journal of Biomechanics, 40(8):1794-805, October 2007.

[35] A. C. Guyton and J. E. Hall. Textbook of Medical Physiology. Elsevier, 2006.

[36] R. P. Franke, R. Fuhrmann, B. Hiebl, and F. Jung. Influence of various radiographic contrast media on the buckling of endothelial cells. Microvascular research, 76(2):110-3, August 2008.

[37] S. K. Sharma. Iodinated contrast media and contrast-induced nephropathy: Is there a preferred cost-effective agent? The journal of invasive cardiology, 20(5):245-8, May 2008.

[38] H. Meng, Z. Wang, Y. Hoi, L. Gao, E. Metaxa, D. D. Swartz, and J. Kolega. Complex hemodynamics at the apex of an arterial bifurcation induces vascular remodeling resembling cerebral aneurysm initiation. Stroke, 38(6):1924-31, June 2007.

[39] S. Periaswamy and H. Faridb. Medical image registration with partial data. Medical Image Analysis, 10(3):452-64, June 2006.

[40] S. Juvela, M. Porras, and K. Poussa. Natural history of unruptured intracranial aneurysms: probability of and risk factors for aneurysm rupture. Journal of Neurosurgery, 108(5):1052-60, May 2008.

[41] S. Dhar, M. Tremmel, J. Mocco, M. Kim, J. Yamamoto, A. H. Siddiqui, L. N. Hopkins, and H. Meng. Morphology parameters for intracranial aneurysm rupture risk assessment. Neurosurgery, 63(2):185-96, August 2008. 